Journal of Fluids and Structures (2000) 000, 000-000

doi:10.1006/jfls.1999.0000, available online at http://www.idealibrary.com on

\title{
Nonlinear synchronization in open flows
}

\author{
Benoît Pier ${ }^{\dagger}$ and PATrick Huerre \\ Laboratoire d'Hydrodynamique (LadHyX), CNRS - École polytechnique, \\ F-91128 Palaiseau cedex, France
}

\begin{abstract}
The selection criteria governing finite-amplitude synchronized oscillating states are discussed for model systems and real wake flows in a domain of infinite streamwise extent. Two types of nonlinear global modes are possible: hat modes with overall smoothly varying amplitude and elephant modes with a sharp front. The vortex street in wake flows is of elephant type, as observed in direct numerical simulations of a real spatially developing wake. Furthermore, the elephant frequency selection criterion is in excellent agreement with the numerically determined vortex shedding frequency.
\end{abstract}

(C) 1999 Academic Press

\section{INTRODUCTION}

A VARIETY OF OPEN FLOWS may sustain globally synchronized oscillations. Wakes behind bluff-bodies are well known (for a review see Williamson 1996) to undergo a transition to a periodic vortex shedding régime at moderate Reynolds numbers. Experimentally and numerically, the features of a globally synchronized vortex street are now well documented (e.g. Provansal et al. 1987, Hammond \& Redekopp 1997). However, the selected frequency of the global structure has not been theoretically predicted in the framework of hydrodynamic stability theory. The aim of the present paper is to discuss recent progress made in the identification of resonance mechanisms which are responsible for global synchronization in spatially developing flows. We restrict here our attention to one-dimensional complex Ginzburg-Landau (CGL) equations with spatially varying coefficients in an infinite domain and to two-dimensional spatially developing wake flows governed by the Navier-Stokes (NS) equations. The paper is mainly based on the recent dissertation of Pier (1999) and the corresponding publications by Pier \& Huerre (1996), Pier et al. (1998), Pier et al. (2001) and Pier \& Huerre (2000). For general background on the hydrodynamic stability theory of spatially developing flows, the reader is referred to the review articles and tutorial presentations of Huerre \& Monkewitz (1990), Huerre \& Rossi (1998) and Huerre (2000). A brief summary of relevant issues is given below.

The existence of self-sustained oscillations in shear flows is closely related to the transition from convective to absolute instability (Briggs 1964; Bers 1983). In convectively unstable (CU) systems, the basic flow carries growing perturbations away in the downstream direc-

† Present address: Department of Applied Mathematics and Theoretical Physics, University of Cambridge, Silver Street, Cambridge CB3 9EW, UK. Email: b.pier@damtp.cam.ac.uk.

0889-9746/99/100813+39 \$30.00/0

(C) 1999 Academic Press 
tion, and the system eventually returns to its unperturbed state. Hence, if a flow changes from local stability to convective instability, only its transient response to perturbations is affected. In a locally stable configuration all perturbations are damped, whereas in a CU basic flow they grow in the downstream direction. In the latter configuration, perturbations eventually leave the domain of interest: in the long term the flow is globally stable and it may only be kept out of equilibrium if perturbations are continuously entering the inflow boundary. Thus CU flows may be interpreted as amplifiers since perturbations entering the inlet grow along the stream until they leave the system at the outlet.

In contrast, transition from convective to absolute instability drastically changes the dynamical behaviour. In absolutely unstable (AU) systems, instabilities grow in situ and survive for all times. A self-sustained non-trivial state may therefore be observed without external input. Thus, as far as the long term asymptotic behaviour is concerned, transition from local stability to convective instability proves irrelevant, whereas transition from convective to absolute instability plays a crucial rôle.

The above mentioned instability properties are defined for infinite and spatially homogenous systems. Since real flows develop in the streamwise direction, stable, CU and AU domains may coexist as the local properties vary along the stream. Under the assumption that the streamwise variations be small on a typical instability length scale, the previous definitions still apply locally at each streamwise station. In wake flows at moderate Reynolds numbers, which are of particular interest to readers of this volume, the transient régime leads to a stationary time-periodic state; the flow globally behaves as an oscillator. Characteristics such as spatial structure and global frequency become intrinsic to the flow: They are selected in the bulk and largely independent of inflow conditions.

The following questions then arise:

1. Under which conditions does global instability occur? Does global instability coincide with the onset of local absolute instability or is an AU domain of finite extent required?

2. In the case of globally synchronized oscillations, how is the global frequency determined? Which part of the flow acts as a wave maker?

3. How is the global behaviour affected by nonlinearities? Are finite-amplitude oscillations governed by linear or nonlinear selection criteria? What is the importance of the mean flow correction generated by nonlinear interactions?

In the following, these issues are preferentially addressed in the context of streamwise developing flows in an infinite domain, whether in the form of CGL model equations or real wakes. In the latter instance we solely consider wakes produced by a velocity deficit introduced at some streamwise station and boldly set aside the wake producing body. This assumption is in marked contrast with the recent investigations by Couairon \& Chomaz $(1997,1999 a, b)$ of nonlinear global modes governed by one-dimensional CGL model equations in semi-infinite domains. In this case, global mode onset takes place whenever a front succeeds in propagating upstream against the advecting flow, thereby getting pinned at the upstream boundary point. This precisely takes place when transition from convective to absolute instability occurs at the upstream boundary. Furthermore, Couairon \& Chomaz (1999b) have derived scaling laws for the global mode characteristic length scale and its streamwise shape near onset which are in excellent agreement with experimental and numerical studies of vortex shedding behind bluff bodies by Goujon-Durand et al. (1994) and Zielinska \& Wesfreid (1995). Such a scenario is also supported by the direct numerical simulations of the nonlinear impulse response in parallel wakes conducted by Delbende \& 
Chomaz (1998): although the impulse response is of finite amplitude, its upstream edge is governed by linear dynamics.

\section{SCALE SEPARATION}

The theoretical formulation underlying all global mode analyses, whether linear or nonlinear, essentially relies on the assumption of slow streamwise variations of instability properties. This hypothesis is required if one is to establish a specific relationship between global behaviour and local properties.

At an intuitive level of understanding, in open systems the mean flow introduces a preferred streamwise direction along which the entire dynamics develops. Let $x$ denote the streamwise distance, increasing from the inlet to the outlet. The coordinate $x$ appears both as a variable in streamwise derivative operators related to the instability properties and as a parameter to account for the streamwise evolution of the basic flow. If $\lambda$ denotes a typical instability length scale and $L$ the streamwise evolution length scale of the basic flow, weak inhomogeneity is characterized by the small parameter

$$
\epsilon \equiv \frac{\lambda}{L} \ll 1
$$

Under assumption (1), the parametric streamwise dependence of the dynamics only occurs through the slow coordinate $X=\epsilon x$. At leading order, the slow parameter $X$ may be considered independent of the fast variable $x$. Local instability characteristics are then retrieved by freezing $X$ in the governing equations and studying the equivalent homogenous system in the infinite domain $-\infty<x<+\infty$. Hence the fast evolving local dynamics is slaved to the slow evolution of the basic flow. This technique is fully justified by resorting to the method of multiple scales (Crighton \& Gaster 1976; Bender \& Orszag 1978).

\section{MODEL FLOWS AND REAL FLOWS}

The global behaviour of spatially developing flows has typically been studied in the context of the one-dimensional CGL model or the complete two-dimensional NS equations.

The spatially inhomogenous Ginzburg-Landau evolution equation for a complex field $\psi(x, t)$ may conveniently be written as

$$
\begin{aligned}
i \frac{\partial \psi}{\partial t}= & \left(\omega_{0}(X)+\frac{1}{2} \omega_{k k}(X) k_{0}(X)^{2}\right) \psi+i \omega_{k k}(X) k_{0}(X) \frac{\partial \psi}{\partial x} \\
& -\frac{1}{2} \omega_{k k}(X) \frac{\partial^{2} \psi}{\partial x^{2}}+\gamma(X)|\psi|^{2} \psi
\end{aligned}
$$

where $\omega_{0}$ and $k_{0}$ denote the absolute frequency and wave number respectively, $\omega_{k k}$ the second derivative of the frequency with respect to wave number. The choice $\gamma_{i} \equiv \operatorname{Im} \gamma<0$ ensures that nonlinearities are stabilizing everywhere. All complex coefficients of equation (2) depend on $X=\epsilon x$ to enforce assumption (1) of weak spatial inhomogeneity.

For two-dimensional incompressible flows, the total streamfunction $\Psi(x, y, t)$ is governed by the non-dimensional vorticity equation

$$
\left(\frac{\partial}{\partial t}+\frac{\partial \Psi}{\partial y} \frac{\partial}{\partial x}-\frac{\partial \Psi}{\partial x} \frac{\partial}{\partial y}\right) \Delta \Psi=\frac{1}{\operatorname{Re}} \Delta^{2} \Psi
$$


where $y$ denotes the cross-stream coordinate and Re the Reynolds number. Real flows fulfill the assumption of slow streamwise development in high Reynolds number situations, and the inhomogeneity parameter then reads

$$
\epsilon=\frac{1}{\mathrm{Re}} .
$$

When $\epsilon \ll 1$, the leading-order time-independent basic flow resulting from (3) obeys the Prantl boundary-layer equation

$$
\left(\frac{\partial \Psi}{\partial y} \frac{\partial}{\partial X}-\frac{\partial \Psi}{\partial X} \frac{\partial}{\partial y}\right) \frac{\partial \Psi}{\partial y}=-\frac{d P}{d X}+\frac{\partial^{3} \Psi}{\partial y^{3}}
$$

where the streamwise pressure gradient $d P / d X$ arises as an integration constant. Basic streamwise and cross-stream velocity components are then obtained as $U(y ; X)=\partial \Psi / \partial y$ and $V(y ; X)=-\epsilon \partial \Psi / \partial X$ respectively. Decomposition of the total streamfunction into basic field and finite-amplitude perturbations according to $\Psi(x, y, t)=\int_{0}^{y} U(\eta ; X) d \eta+\psi(x, y, t)$ yields the governing vorticity equation for $\psi(x, y, t)$ as

$$
\left(\frac{\partial}{\partial t}+U(y ; X) \frac{\partial}{\partial x}\right) \Delta \psi-\frac{\partial^{2} U}{\partial y^{2}}(y ; X) \frac{\partial \psi}{\partial x}+\left(\frac{\partial \psi}{\partial y} \frac{\partial}{\partial x}-\frac{\partial \psi}{\partial x} \frac{\partial}{\partial y}\right) \Delta \psi=\frac{1}{\operatorname{Re}} \Delta^{2} \psi .
$$

Although real flows are two-dimensional, there is only one slow streamwise coordinate $X$ that accounts for the evolution of local instability properties as described by a local dispersion relation. The study of two-dimensional flows is more complex since cross-stream eigenfunctions have to be computed; however the analysis of CGL or NS global modes proceeds in exactly the same manner since all the fast evolving features are slaved to $X$.

Note that in the CGL model (2) any variations of the complex coefficients with $X$ may be considered. In the hydrodynamic context (6) however, the basic flow $U(y ; X)$ governed by (5) is uniquely determined by the inlet velocity profile, say $U(y ; X=0)$, and the streamwise pressure distribution $P(X)$ for $X>0$. In a self-consistent formulation, the velocity profiles $U(y ; X)$ appearing as coefficients in (6) cannot be arbitrarily specified. In the present analysis, a coflowing wake profile is chosen for $U(y ; X=0)$ and the pressure field $P(X)$ is then carefully tailored so that the essential features of experimental wake flows are reproduced. In this procedure, a "synthetic wake" is thereby generated without requiring the presence of a solid obstacle (cf. Figure 2a)!

\section{LINEAR RESONANCE CRITERION}

In a stricly linear framework, theoretically consistent results have first been derived by Chomaz et al. (1991) for the linear version of model (2) and by Monkewitz et al. (1993) for the linear version of the vorticity equation (6). The essential physical property is the complex local absolute frequency $\omega_{0}(X)$ defined in classical fashion (Briggs 1964; Bers 1983) by imposing a zero group velocity condition on the local linear dispersion relation

$$
\omega=\Omega^{\ell}(k, X) .
$$

The form of the CGL model (2) already displays its dependence on $\omega_{0}(X)$, whereas for real flows the dispersion relation (7) is derived by solving the Orr-Sommerfeld equation applied to the velocity profiles $U(y ; X)$ prevailing at each station $X$. 

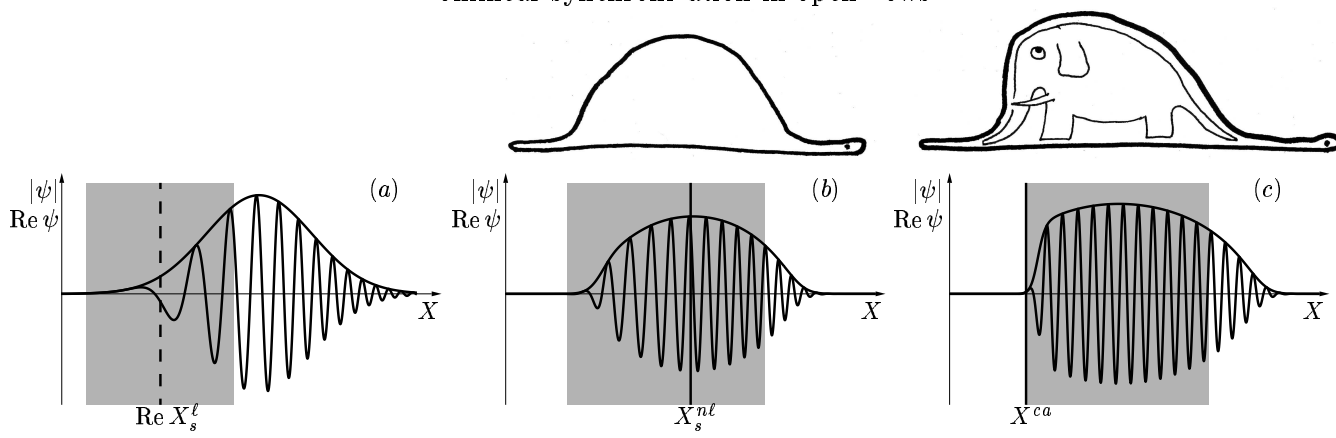

Figure 1. Shapes of CGL global modes. Shaded regions indicate extent of AU domain. (a) Linear global mode. (b) Nonlinear soft global mode or hat mode. (c) Nonlinear steep global mode or elephant mode. The names "hat" and "elephant" have been chosen in reference to Saint-Exupéry (1946).

The criterion for linear global instability is then based on the variations of $\omega_{0}(X)$ and states that the complex frequency $\omega_{s}^{\ell}$ of a self-sustained linear global mode is given by the saddle-point condition

$$
\frac{d \omega_{0}}{d X}\left(X_{s}^{\ell}\right)=0 \quad \text { and } \quad \omega_{s}^{\ell}=\omega_{0}\left(X_{s}^{\ell}\right),
$$

where it is understood that $\omega_{0}(X)$ has been analytically continued in the complex $X$-plane. In general, the saddle point $X_{s}^{\ell}$ does not occur on the real axis, and linear global instability characterized by $\operatorname{Im} \omega_{s}^{\ell}>0$ requires an AU region of finite extent in the slow variable $X$. Thus, in the linear framework, absolute instability is a prerequisite for global instability. However it is not a sufficient condition: Linear global modes are observed to decay in time for AU domains of finite but small extent in $X$, which may correspond to very large AU domains in terms of $x$.

The typical shape of a linear CGL global mode is sketched in Figure 1(a). Note that, in general, maximum amplitude occurs downstream of $\operatorname{Re} X_{s}^{\ell}$.

\section{NONLINEAR RESONANCE CRITERIA}

A weakly nonlinear approach (Le Dizès et al. 1993) conducted close to the onset of global instability specified by $\operatorname{Im} \omega_{s}^{\ell}=0$ has proven that the bifurcation analysis is ill-behaved and suggested that only a fully nonlinear theory is appropriate. In the nonlinear framework, two types of finite-amplitude oscillating states have been identified for the inhomogenous CGL equation in infinite media: soft or hat (Pier \& Huerre 1996) and steep or elephant (Pier et al. 1998) nonlinear global modes (cf. Figure $1 b, c)$. Their selection criteria are obtained from the local linear and nonlinear dispersion relations, as summarized below.

The local nonlinear dispersion relation is defined via a temporal evolution problem in the following way. Consider a homogenous medium obtained by freezing $X$ at a prescribed value. An unstable spatially periodic perturbation of real wave number $k$ grows according to $\Omega_{i}^{\ell}(k, X)>0$ until its amplitude reaches a finite level. Due to stabilizing nonlinearities, a fully nonlinear wavetrain is generated with spatial periodicity imposed by the initial wave number. Its frequency, measured for each $k$, then yields the nonlinear dispersion relation 


$$
\omega=\Omega^{n \ell}(k, X) .
$$

Whereas the local linear dispersion relation (7) yields a complex frequency for any complex wave number, the nonlinear dispersion relation (9) is defined only for real wave numbers $k$ associated with a positive growth $\operatorname{rate} \Omega_{i}^{\ell}(k, X)>0$ and it necessarily yields real frequencies. For the CGL model (2) nonlinear wave trains are finite-amplitude harmonic waves of the form $R e^{i(k x-\omega t)}$ and (9) reads $\omega=\operatorname{Im}\left(\gamma^{\star}(X) \Omega^{\ell}(k, X)\right) / \operatorname{Im} \gamma^{\star}(X)$. Computation of (9) for real flows, however, requires a numerical integration as discussed by Pier \& Huerre (2000).

Hat global modes (Figure 1b; Pier \& Huerre 1996) have an overall smoothly varying amplitude, and their real frequency $\omega_{s}^{n \ell}$ is selected at a saddle-point $X_{s}^{n \ell}$ of the nonlinear dispersion relation according to

$$
\frac{\partial \Omega^{n \ell}}{\partial X}\left(X_{s}^{n \ell}, k_{s}^{n \ell}\right)=\frac{\partial \Omega^{n \ell}}{\partial k}\left(X_{s}^{n \ell}, k_{s}^{n \ell}\right)=0 \quad \text { and } \quad \omega_{s}^{n \ell}=\Omega^{n \ell}\left(k_{s}^{n \ell}, X_{s}^{n \ell}\right) .
$$

Elephant global modes (Figure 1c; Pier et al. 1998) are characterized by a sharp front governed by the Dee \& Langer (1983) marginal stability criterion and located at the upstream transition station $X^{c a}$ between $\mathrm{CU}$ and $\mathrm{AU}$ regions. The entire structure is tuned to the front frequency given by the corresponding real absolute frequency

$$
\omega_{0}^{c a}=\omega_{0}\left(X^{c a}\right) \quad \text { with } \quad \operatorname{Im} \omega_{0}\left(X^{c a}\right)=0 .
$$

The detailed analysis of the transition scenarii between the unperturbed state and either type of nonlinear global mode reveals (Pier 1999; Pier et al. 2001) that the two nonlinear resonance criteria $(10,11)$ are mutually exclusive and that the appropriate global bifurcation parameter is the maximum absolute growth rate over the entire medium $\omega_{0, i}^{\max } \equiv$ $\max \omega_{0, i}(X)$. Nonlinear global modes exist whenever an AU region is present $\left(\omega_{0, i}^{\max }>0\right)$. At transition $\left(\omega_{0, i}^{\max }=0\right)$ an elephant mode is always selected. Hat modes exist further above threshold and are more readily obtained in systems where the basic advection velocity is weak. Absolute instability is therefore a necessary and sufficient condition for the existence of self-sustained nonlinear structures. This is in contrast with the results of section 4: Local absolute instability is only a necessary condition for the existence of amplified linear global modes.

\section{FINITE-AMPLITUDE VORTEX STREET AS AN ELEPHANT MODE}

The generalization of the above nonlinear theory to real flows governed by the NavierStokes equations has been conducted by Pier (1999) and Pier \& Huerre (2000).

In order to obtain unambiguous results that can be compared with the theory, the basic flow has to strictly comply with the condition of weak streamwise nonuniformity. Bluff body wakes display a recirculation bubble near the obstacle which violates this assumption. The "synthetic wakes" governed by the Prandtl boundary-layer equation (5) all avoid this difficulty. In the example represented in Figure $2(a)$ for $R e=100$, the pressure gradient has been selected to be mildly adverse in order to produce a central AU region (displayed in gray), which is an essential feature of real wakes.

Direct numerical simulations of the temporal evolution of this basic flow (see Pier \& Huerre (2000) for details) leads to a finite-amplitude vortex shedding régime (Figure $2 b$ ) tuned at a well defined global frequency $\omega_{g}=0.186 \pm 0.002$. This vortex street is made 

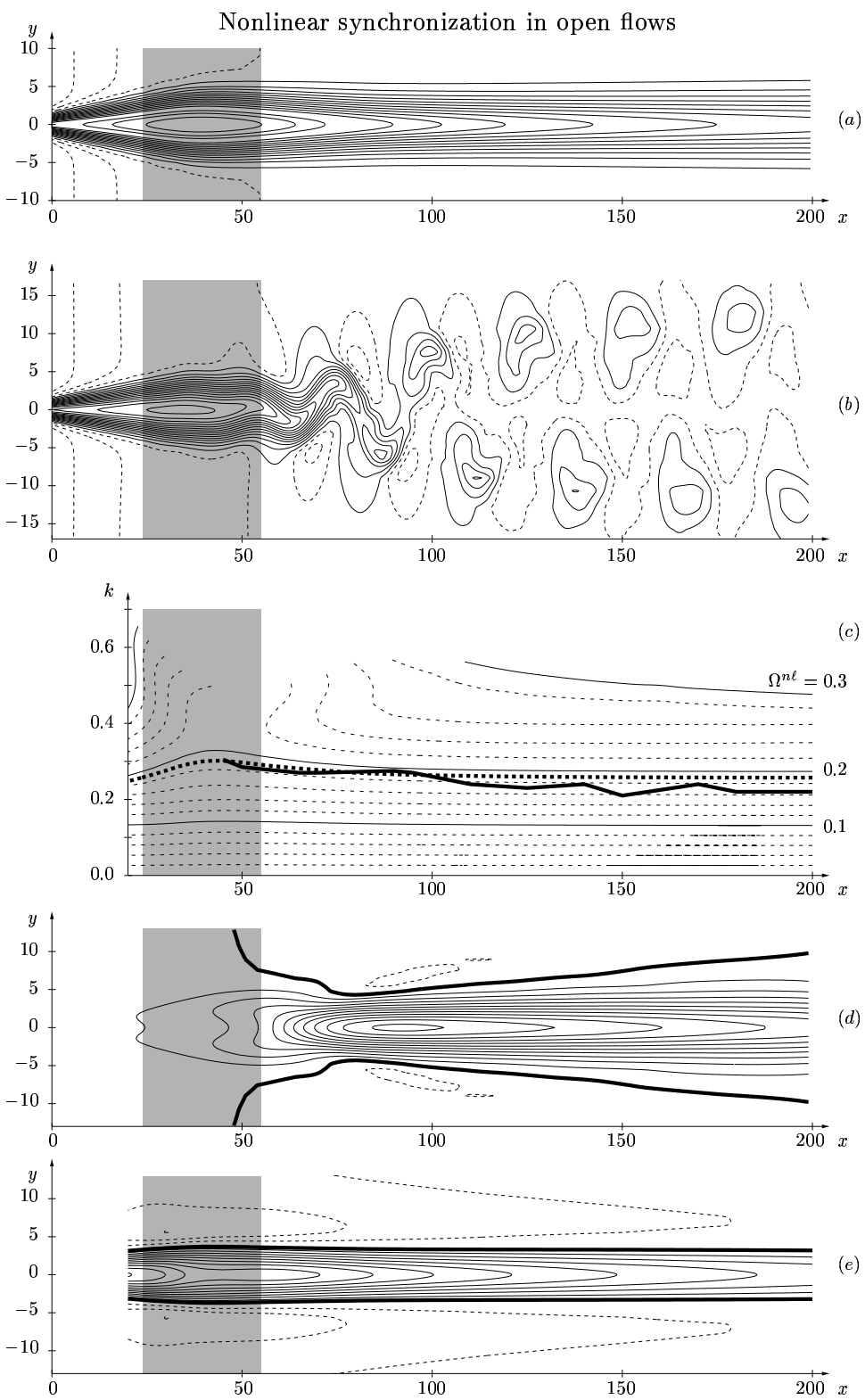

Figure 2. Intrinsic synchronization of spatially developing wake flow at $R e=100$. Shaded regions indicate extent of AU domain. (a) Streamwise velocity isolines of unperturbed basic wake flow. (b) Snapshot of total streamwise velocity isolines in periodic vortex shedding régime. $(a, b)$ Dashed lines represent the levels $0.95,0.90,0.85$ above the outlet free-stream velocity; solid lines pertain to the levels $0.80, \ldots, 0.10$. (c) Isofrequency contours of the nonlinear dispersion relation $\Omega^{n \ell}(k, X)$ in the linearly unstable domain of the $(X, k)$-plane. Thin solid contours are separated by $\delta \Omega=0.1$, thin dashed contours by $\delta \Omega=0.02$. Predicted nonlinear spatial branch $k^{n \ell}\left(X, \omega_{0}^{c a}\right)$ of global frequency $\omega_{0}^{c a}=0.19$ is represented by thick dashed curve. Observed local wave number in $(b)$ follows path indicated by thick solid line. $(d)$ Mean streamwise velocity correction of vortex street sketched in $(b)$. (e) Mean streamwise velocity correction of local nonlinear wavetrains of wave number $k^{n \ell}\left(X, \omega_{0}^{c a}\right)$. $(d, e)$ Thick line separates regions of positive $(0.05,0.10, \ldots$ solid lines $)$ and negative $(-0.05,-0.10, \ldots$ dashed line $)$ levels. 
up of wavetrains which slowly deform while travelling downstream: such structures are locally periodic and their $(x, t)$-dependence solely occurs via a phase function with only slow streamwise variations. Hence the multiple-scale formalism (Bender \& Orszag 1978) applies and the global structure may be analysed in terms of local linear and nonlinear waves.

In the upstream domain $(x<25)$, the basic flow is seen to remain unperturbed: smallamplitude wavetrains prevail in this linear region. Further downstream, nonlinear travelling waves develop and completely mask the underlying basic wake flow. In order to establish that the nonlinear globally synchronized state (Figure $2 b$ ) follows the elephant resonance criterion (11), its numerically determined features are now compared with predictions based on the local linear and nonlinear instability analyses of the basic flow (Figure $2 a$ ).

The computation of the local linear dispersion relation (7) via the Orr-Sommerfeld equation reveals an $\mathrm{AU}$ domain extending over the streamwise interval $24<x<55$ (gray regions in Figure 2). The real absolute frequency prevailing at its upstream boundary $x^{c a}=24$ reads $\omega_{0}^{c a}=0.190$. The nonlinear resonance criterion (11) therefore accurately predicts the vortex shedding frequency, unlike the linear criterion (8) which yields $\omega_{s}^{\ell}=0.143+0.008 i$.

The local nonlinear dispersion relation (9) is illustrated in Figure 2(c) by isofrequency contours in the linearly unstable domain of the $(X, k)$-plane. These contours precisely define the nonlinear spatial branches $k^{n \ell}(X, \omega)$ obtained by solving the nonlinear dispersion relation (9) at a given frequency. A global mode synchronized at the frequency $\omega_{0}^{c a}$ is expected to follow the spatial branch $k^{n \ell}\left(X, \omega_{0}^{c a}\right)$ represented by a thick dashed curve. The local wave number of the numerically computed spatially developing vortex street (Figure $2 b$ ) is represented by a thick solid curve in the same sketch and it is seen to closely follow the path predicted by the elephant global mode structure.

The finite-amplitude vortex street is thus described by a nonlinear elephant global mode. This theory not only accurately predicts the vortex shedding frequency but also the spatial structure of the downstream developing vortex street.

The mean-flow correction, which is absent in CGL models, is specific to real shear flows. Indeed, nonlinear quadratic interactions in the NS equations generate a time-independent mean-flow component as well as higher harmonics. In the fully developed vortex shedding régime, the total mean flow then results from the superposition of the basic flow (Figure 2a) and the mean-flow correction (Figure $2 d$ ). According to Figure 2(d), the mean-flow correction tends to fill up the velocity deficit in the wake. It is instructive to compare the results of direct numerical simulations with those emerging from a temporal evolution problem pertaining to a parallel wake frozen at a prescribed $X$ station and perturbed with a spatially periodic wave of wavenumber $k^{n \ell}\left(X, \omega_{0}^{c a}\right)$. Via this procedure, a finite-amplitude wavetrain is obtained for large time, the frequency of which is precisely $\omega_{0}^{c a}$. Local mean-flow corrections are thereby computed for each station $X$, which may be pieced together to generate a spatially evolving mean correction field as displayed in Figure 2(e). The agreement between direct numerical simulation (Figure $2 d$ ) and local predictions (Figure $2 e$ ) is less satisfactory than for the unsteady part of the flow field (Figure $2 c$ ). Whereas in the limit of vanishing inhomogeneity, the local analysis predicts a mean-flow correction of almost constant cross-stream width, the width of the mean flow in the direct numerical simulation is seen to increase with downstream distance. The mean-flow correction field is generated in the central shear region by nonlinear interactions and slowly diffuses on a viscous scale into the outer cross-stream direction. This diffusion process takes place in time (Figure $2 e$ ) or along the stream (Figure 2d), and there is no obvious relationship between these two situations. 


\section{CONCLUSIONS}

We are now in a position to answer the questions listed in the introductory section.

1. In a strictly linear approximation global instability in general requires an AU region of finite extent, whereas nonlinear global instability takes place as soon as local absolute instability arises at some point in the flow. When nonlinearities are present, the linear resonance criterion (8) becomes irrelevant. It is the existence of a transition point from convective to absolute instability which is crucial in the establishment of a self-sustained nonlinear state. Note that the real cylinder wake becomes absolutely unstable at $\operatorname{Re} \sim 25$ whereas onset of vortex shedding occurs only for Re $\sim 46$. This discrepancy might be due to a violation of the assumption of slow spatial development in the neighborhood of the obstacle or to a non-trivial effect of the solid boundary.

2. The complex frequency of a linear global mode (Figure 1a) is obtained at a saddle point $X_{s}^{\ell}$ of $\omega_{0}(X)$ analytically continued in the complex $X$-plane (8). Due to this continuation procedure, no frequency generating location may be identified in physical space; note however that the region of maximum absolute growth rate plays an essential part. In contrast, the global frequency of nonlinear global modes (Figure $1 b, c$ ) is selected at a specific location: either the saddle point $X_{s}^{n \ell}$ of the nonlinear dispersion relation (10) or the upstream boundary $X^{c a}$ of the AU region (11). These nonlinear resonance criteria are purely local in the sense that only the properties of the system at these stations are involved. In the case of wake flows, the vortex street is triggered by a front structure at $X^{c a}$ which acts as a source and imposes its frequency to the entire flow.

3. The selection mechanisms pertaining to hat and elephant nonlinear global modes are markedly distinct. The hat frequency selection criterion (10) involves a saddle point of the nonlinear dispersion relation (9) in the bulk of the finite-amplitude region (Figure 1b). Elephant modes (Figure 1c) are selected by a front located at the upstream boundary of the AU domain; finite amplitude wavetrains develop downstream of this location. Since the elephant frequency selection criterion (11) only involves the linear dispersion relation, this variety of nonlinear global mode is surprisingly governed by a local linear criterion.

Finite-amplitude vortex shedding in wakes generates a mean flow correction comparable in magnitude to the basic flow. Nonlinearities thus completely modify the underlying basic flow which becomes unobservable unless one artificially kills the perturbations by imposing for example a symmetry condition.

The comparison between the results of direct numerical simulations and locally computed nonlinear wavetrains has demonstrated the validity of a linear and nonlinear analysis based on a scale separation assumption. The theory has led to the identification of two varieties of global modes: elephants and hats. The vortex street has been shown to be of elephant type with a front located at the convective-absolute instability transition point imposing its frequency to the entire flow. There remains to determine a real flow that sustains a global mode of hat-type. Rayleigh-Bénard convection in the presence of a horizontally varying temperature difference or Taylor-Couette flow between rotating coaxial cylinders with a varying gap may be good candidates for such a situation since there is no basic advection.

\section{REFERENCES}

Bender, C. M. \& ORszag, S. A. 1978 Advanced Mathematical Methods for Scientists and Engineers. New York: McGraw-Hill. 
BERS, A. 1983 Space-time evolution of plasma instabilities - absolute and convective. In Handbook of Plasma Physics (eds. M. N. Rosenbluth \& R. Z. Sagdeev), pp. 451-517, Amsterdam: NorthHolland.

BRIgGs, R. J. 1964 Electron-Stream Interaction with Plasmas. Cambridge, Mass.: M.I.T. Press.

Chomaz, J.-M., HuerRe, P. \& REDEKopP, L. G. 1991 A frequency selection criterion in spatially developing flows. Studies in Applied Mathematics 84, 119-144.

CouAiron, A. \& Chomaz, J.-M. 1997 Absolute and convective instabilities, front velocities and global modes in nonlinear systems. Physica D 108, 236-276.

CouAiron, A. \& Chomaz, J.-M. 1999a Primary and secondary nonlinear global instability. Physica $D$ 132, 428-456.

CouAiron, A. \& Chomaz, J.-M. 1999b Fully nonlinear global modes in slowly varying flows. Physics of Fluids 11, 3688-3703.

CRIGhton, D. \& Gaster, M. 1976 Stability of slowly diverging jet flow. Journal of Fluid Mechanics 77, 397-413.

DEE, G. \& LANGER, J.S. 1983 Propagating pattern selection. Physical Review Letters 50, 383-386.

Delbende, I. \& Chomaz, J.-M. 1998 Nonlinear convective/absolute instabilities in parallel twodimensional wakes. Physics of Fluids 10, 2724-2736.

Goujon-Durand, S., Jenffer, P. \& Wesfreid, J. E. 1994 Downstream evolution of the Bénardvon Kármán instability. Physical Review E 50, 308-313.

HAMMOND, D. \& REDEKOPP, L. 1997 Global dynamics of symmetric and asymmetric wakes. Journal of Fluid Mechanics 331, 231-260.

HUERRE, P. 2001 Open shear flow instabilities. To appear in Perspectives in Fluid Dynamics (eds. G. K. Batchelor, H. K. Moffatt \& M. G. Worster), Cambridge: Cambridge University Press.

HuerRe, P. \& Monkewitz, P. A. 1990 Local and global instabilities in spatially developing flows. Annual Review of Fluid Mechanics 22, 473-537.

HueRRE, P. \& Rossi, M. 1998 Hydrodynamic instabilities in open flows. In Hydrodynamics and Nonlinear Instabilities (eds. C. Godrèche \& P. Manneville), pp. 81-294, Cambridge: Cambridge University Press.

Le Dizès, S., Huerre, P., Chomaz, J.-M. \& Monkewitz, P. A. 1993 Nonlinear stability analysis of slowly-diverging flows: limitations of the weakly nonlinear approach. In Proceedings of the IUTAM Symposium on Bluff-Body Wakes, Dynamics and Instabilities (eds. H. Eckelmann, J. M. R. Graham, P. Huerre \& P. A. Monkewitz), pp. 147-152, Berlin: Springer.

Le Dizès, S., Huerre, P., Chomaz, J.-M. \& Monkewitz, P. A. 1996 Linear global modes in spatially developing media. Philosophical Transactions of the Royal Society (London) A354, $169-212$

Monkewitz, P. A., Huerre, P. \& Chomaz, J.-M. 1993 Global linear stability analysis of weakly non-parallel shear flows. Journal of Fluid Mechanics 251, 1-20.

PIER, B. 1999 Comportement non linéaire synchronisé dans les écoulements cisaillés. Ph.D. Dissertation, École polytechnique, Palaiseau, France.

PIER, B. \& HUERRE, P. 1996 Fully nonlinear global modes in spatially developing media. Physica $D$ 97, 206-222.

PIER, B. \& HUERRE, P. 2000 Nonlinear self-sustained structures and fronts in wake flows. submitted to Journal of Fluid Mechanics.

Pier, B., HuerRe, P. \& CHOMAZ, J.-M. 2001 Bifurcation to fully nonlinear synchronized structures in slowly varying media. Physica D 148, 49-96.

Pier, B., Huerre, P., Chomaz, J.-M. \& CouAiron, A. 1998 Steep nonlinear global modes in spatially developing media. Physics of Fluids 10, 2433-2435.

Provansal, M., MAThis, C. \& BOYER, L. 1987 Bénard-von Kármán instability: transient and forced regimes. Journal of Fluid Mechanics 182, 1-22.

SAINT-ExUPÉRY, A. DE 1946 Le Petit Prince. Paris: Gallimard.

Williamson, C. H. K. 1996 Vortex dynamics in the cylinder wake. Annual Review of Fluid Mechanics 28, 477-539.

ZIELINSKA, B. J. A. \& WESFREID, J. E. 1995 On the spatial structure of global modes in wake flow. Physics of Fluids 7, 1418-1424. 